\title{
41. Early clinical experience with cluster resection and transplantation for right upper quadrant abdominal malignancy
}

\author{
ROBERT D. GORDON, SATORU TODO, ANDREAS G. TZAKIS and \\ THOMAS E. STARZL
}

Liver transplantation for primary tumors originating in the liver or biliary tree, not suitable because of location of extent for subtotal hepatic resection, has been disappointing. So has liver transplantation for tumors metastatic to the liver. Although many patients receiving a liver transplant for malignant disease are technically easier to operate on than patients with cirrhosis and such patients often make a swift postoperative recovery, disease recurrences within 6 to 18 months is common despite the additional field of resection a transplant hepatectomy can provide. Patients with disease extending beyond the liver capsule, into regional lymph nodes, or into major vascular structures such as the portal vein or vena cava, have a particularly poor prognosis and are probably not suitable candidates for liver transplantation alone.

New approaches are needed if patients with right upper quadrant abdominal malignancies are to be helped. Either additional surgical resection or additional modes of chemotherapy or biological modification may be needed. Actually, a combination of innovative surgical and medical techniques may be needed.

The liver and pancreas develop as outpouchings of the foregut region which ultimately becomes the duodenum. Tumors arising in one of these organs tend to spread into one of the other organs or to the transverse mesocolon or colon. This prompted our transplant surgeons to consider whether a more radical en-bloc excision of the right upper quadrant viscera, including the liver, duodenum, pancreas, right colon, and all or part of the stomach, and the associated regional lymphatic drainage, followed by liver or liver-pancreas replacement, might be of benefit in the management of right upper quandrant abdominal cancer.

Starzl long ago realized the surgical feasibility of the solid organ abdominal cluster transplant (1). In 1962 he published a description of transplantation in the dog of an abdominal organ cluster, including the liver, stomach, spleen, pancreas, small bowel and colon based on an aortic stalk (Figure 1). Venous return was provided by reanastomosis of the infrahepatic and suprahepatic vena cava. This early experience was finally applied to clinical practice 27 years later when in 1987 a child with short gut syndrome and hepatic failure was successfully transplanted in Pittsburgh and survived for 6 months (2). The current 


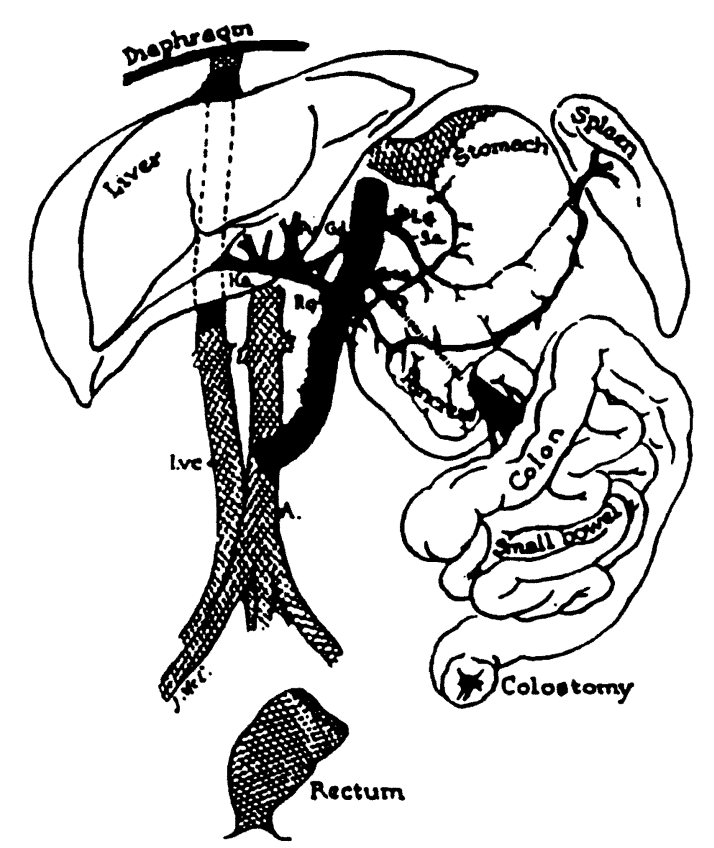

Fig 1. Starzl's original experimental approach to the abdominal cluster organ transplant in the dog. The abdominal viscera are based on an aortic stalk reimplanted end-to-side to the recipient aorta. The only venous reconstruction required is the supra- and infra-hepatic vena cava. (From reference (1).)

surgical methods used for organ cluster resection and transplantation (Figure 2) and our earliest results were recently reported by Starzl et al. (3). A modification of the procedure in which liver transplantation alone (Figure 3 ) is used to replace the resected organs was recently described by Tzakis et al. (4). Subsequent experience with the first 34 patients to undergo such a procedure is summarized in Table 1 . Seventeen patients were given a liver transplant after upper abdominal organ cluster resection and 17 received a composite liverpancreas graft (organ cluster graft).

Sixteen of the original 34 patients are surviving 4-9 months after operation. Four survivors are known to have recurrent disease. Thus, there are $12(35.3 \%)$ survivors presently believed to be disease free. Serious technical complications have most often been related to complications of the pancreatic portion of the graft and have included pancreatitis, abscess, sepsis, and mycotic aneurysm. For this reason, we have used simple liver replacement and pancreatic enzyme and insulin replacement therapy in later parts of our experience. Malnutrition associated with total pancreatectomy or the extensive upper GI tact resection, has been the most significant cause of morbidity in surviving patients.

A few of the patients with early recurrence of cancer were probably inaccurately staged and may have already had microscopically disseminated tumor 


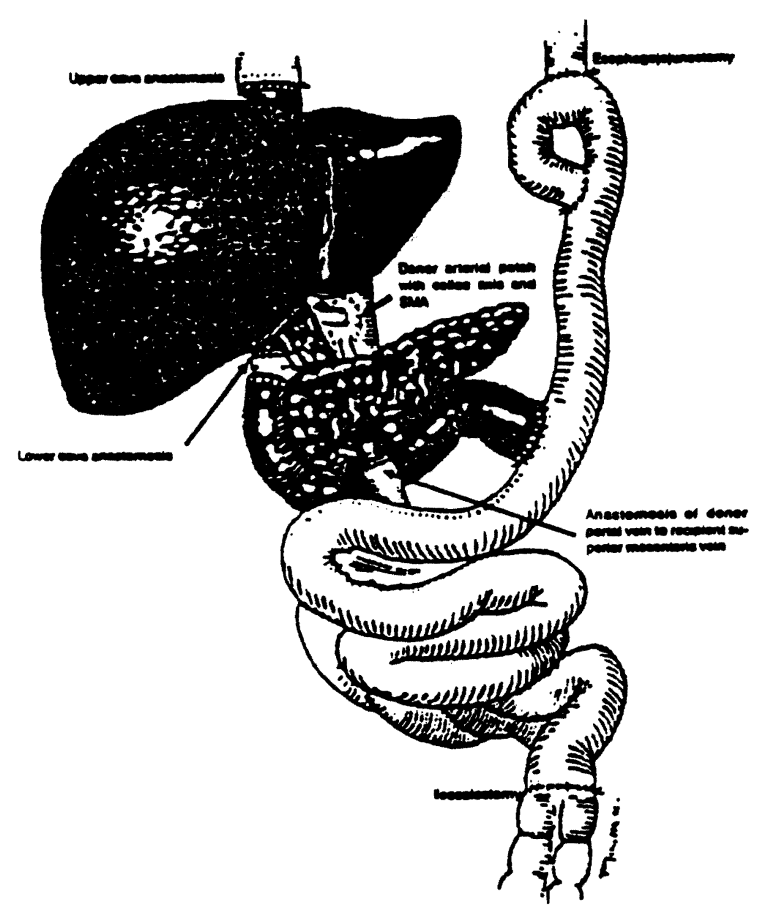

Fig 2. The organ cluster transplant for replacement of vital abdominal organs after right upper quadrant exenteration (organ cluster resection). In the operation shown, the arterial reconstruction is a Carrel patch of donor aorta containing both the celiac axis and the superior mesenteric artery to preserve blood supply to both the liver and pancreas. The venous reconstruction requires anastomosis of the donor portal vein to the recipient superior mesenteric vein and the supra- and infrahepatic vena cava anastomoses. Biliary reconstruction is not needed. (Adapted from reference (3).)

beyond the bounds of resection prior to operation. Clearly accurate staging of disease is important before undertaking such surgery and new technology may need to be applied to accomplish this. Nevertheless, although our experience is limited and follow-up for most of the patients is relatively short, we have a small but encouraging set of patients surviving beyond 6 months after surgery who are out of the hospital and so far appear free of cancer.

At the present time, this unique approach to the management of conventionally unresectable cancer must still be considered experimental. We are still learning how to best stage these patients for surgery, which tumors are best suited for this type of management, what adjunctive therapy is indicated, and how to minimize the technical complications and reduce the morbidity of the procedure. The use of cluster resection with liver replacement only has eliminated the complications associated with intraabdominal pancreas transplantation, but has added diabetes and nutritional complications. Increased surgical experience has already reduced the incidence and severity of technical com- 


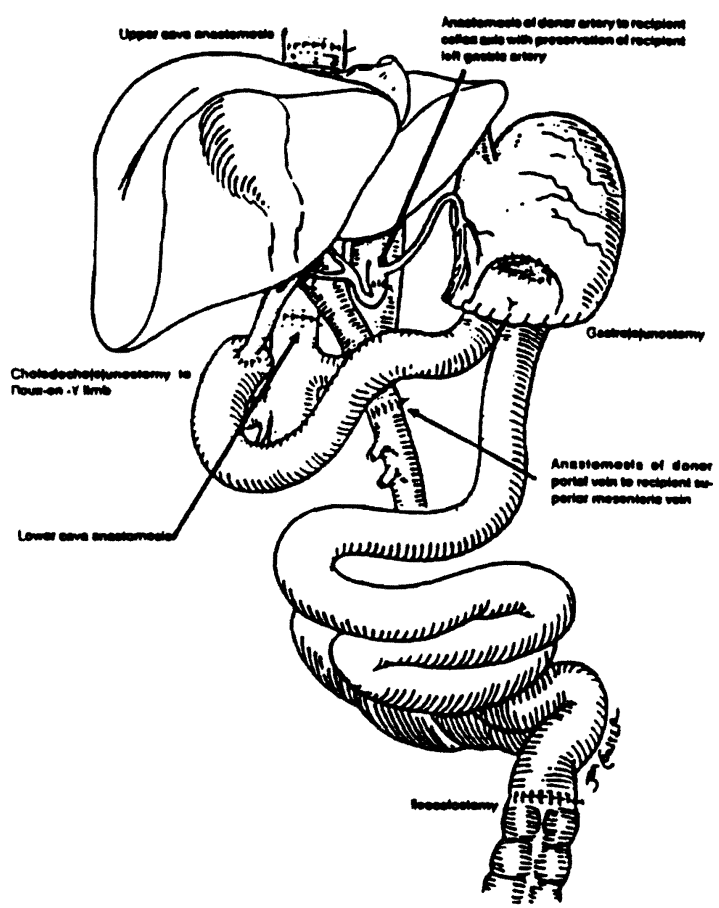

Fig 3. Liver transplantation (without cluster) for reconstruction after right upper quadrant exenteration (organ cluster resection). In the procedure shown, the proximal celiac axis and left gastric artery of the recipient have been retained which permits preservation of the proximal stomach. (Adapted from reference (4).)

Table 1 . Outcome after cluster resection and transplantation for right upper quadrant malignancy (4 to 9 months follow-up).

\begin{tabular}{llr}
\hline Indication for resection & Alive & Dead \\
\hline Cholangiocarcinoma & $9(3)$ & 10 \\
Hepatocellular carcinoma & $2(1)$ & 4 \\
Carcinoid tumor & $2(0)$ & 1 \\
Spindle cell sarcoma & $1(0)$ & 1 \\
Neuro-endocrine tumor & $2(0)$ & 0 \\
Islet cell tumor & 0 & 1 \\
Pancreatic cancer & & 1 \\
Total & $16(4)$ & 18 \\
\hline
\end{tabular}

plications in subsequently treated patients. The use of home hyperalimentation for a prescribed period is being tried and may help with management of the early nutritional problems. 


\section{Acknowledgement}

This work was supported by research grants from the Veterans Administration and Project Grant No. DK29961 from the National Institutes of Health, Bethesda, MD.

\section{References}

1. Starzl, T.E.. Kaupp, H.A.. Brock. D.R. et al.: Homotransplantation of multiple abdominal viscera. Amer. J. Surg. 103.219-229 (1962).

2. Starzl. T.E., Rowe, M.I., Todo, S. et al.: Transplantation of multiple abdominal viscera. $J$. Amer. Med. Assoc. 261, 1449-1457 (1989).

3. Starzl, T.E.. Todo, S., Tzakis. A.G. et al.: Abdominal organ cluster transplantation for the treatment of upper abdominal malignancies. Ann. Surg. 210, 118-130 (1989).

4. Tzakis, A.G.. Todo, S., and Starzl. T.E.: Upper abdominal exenteration with liver replacement: a modification of the cluster procedure. Transplant. Proc. 22 273-274 (1990). 